\title{
FRUTIFICAÇÃO PRECOCE DE LARANJEIRAS 'MONTE PARNASO' COM ANELAGEM E PULVERIZAÇÕES DE ÁCIDO GIBERÉLICO E ÓLEO MINERAL
}

\author{
OTTO CARLOS KOLLER ${ }^{2}$, FERNANDO FERRARI SOBRINHO ${ }^{3}$ e SERGIO FRANCISCO SCHWARZ ${ }^{4}$
}

\begin{abstract}
RESUMO - Com o objetivo de diminuir a floração muito intensa e aumentar a produção de frutos, laranjeiras de umbigo 'Monte Parnaso' (Citrus sinensis Osb.), com dois anos de idade, foram submetidas à anelagem de casca do tronco, pulverizações foliares com doses de $0,10,20$ e $30 \mathrm{ppm}$ de ácido giberélico $\left(\mathrm{AG}_{3}\right)$, combinadas com 0 e $1 \%$ de óleo mineral emulsionável. Foram realizados dois experimentos: um com pulverizações repetidas em 22 e 29/07/93, e outro com pulverização em plena floração em 15/09/93. Em ambos os experimentos, $50 \%$ das plantas foram submetidas à anelagem da casca do tronco, feita por uma incisão anelar, $5 \mathrm{~cm}$ abaixo da formação da copa. A produção diminuiu linearmente com o aumento das dosagens de $\mathrm{AG}_{3}$ pulverizado no final de julho; não houve efeito significativo sobre a frutificação quando o $\mathrm{AG}_{3}$ foi aplicado em plena floração. As pulverizações com $1 \%$ de óleo mineral diminuíram o número de frutos produzidos, em até $42 \%$, principalmente nos tratamentos feitos no final de julho. A anelagem da casca do tronco aumentou o número de frutos em aproximadamente $25 \%$, mas diminuiu o peso médio dos frutos.
\end{abstract}

Termos para indexação: Citrus sinensis, fixação de frutos, produtividade.

\section{EFFECT OF RINGING AND SPRAYING WITH GIBBERELLIC ACID AND MINERAL OIL ON THE FRUITING OF YOUNG 'MONTE PARNASO' NAVEL ORANGE TREES}

\begin{abstract}
Aiming to study fruit set increase of young 'Monte Parnaso' navel orange trees (Citrus sinensis Osb.), plants were sprayed with $0,10,20$, or $30 \mathrm{ppm}$ of gibberellic acid $\left(\mathrm{GA}_{3}\right)$ solution, plus 0 or $1 \%$ of mineral oil. Two experiments were set up: one spraying the trees twice, on $22^{\text {nd }}$ and $29^{\text {th }}$ of July, 1993, and another spraying the trees on $15^{\text {th }}$ of September, 1993, when it was all at full bloom. In both experiments $50 \%$ of the trees were ringed $5 \mathrm{~cm}$ down the first branch of the crown. Results showed that fruit production decreased linearly with the increasing of GA, applyied on $22^{\text {nd }}$ and $27^{\text {th }}$ of July, 1993; there was no significant effect for $\mathrm{GA}_{3}$ applyied at full bloom. The spraying of $1 \%$ mineral oil decreased harvest by $42 \%$, mainly when applyied at the end of July. Ringing increased fruiting nearly to $25 \%$ and decreased the average fruit size.
\end{abstract}

Index terms: Citrus sinensis, fruit set, productivity.

\section{INTRODUÇÃO}

As laranjas de umbigo (Citrus sinensis Osb.) são muito valorizadas no mercado de fruta fresca. Aliado às características de qualidade, a laranjeira de umbigo 'Monte Parnaso' produz frutos tardios que, no

\footnotetext{
${ }^{1}$ Aceito para publicação em 29 de junho de 1998. Pesquisa financiada pelo CNPq, FAPERGS e FINEP.

${ }^{2}$ Eng. Agr, Dr., Prof. Adjunto, Faculdade de Agronomia, UFRGS, Caixa Postal 776, CEP 91501-970 Porto Alegre, RS. Bolsista do CNPq. E-mail: ockoller@myway.com.br

${ }^{3}$ Eng. Agr., UFRGS. Bolsista da FAPERGS

${ }^{4}$ Eng. Agr., UFRGS
}

Rio Grande do Sul, são colhidos nos meses de agosto, setembro e outubro, valorizando ainda mais o seu preço.

A laranjeira 'Monte Parnaso', entretanto, apresenta uma produtividade muito baixa, geralmente atingindo apenas $1 / 3$ a 1/4 da alcançada pela laranjeira 'Valência' (Koller, 1993).

A causa principal dessa baixa produtividade pode ser atribuída a desequilíbrios nutricionais e hormonais relacionados com a ausência de sementes e intensa floração, em cachos florais desprovidos de folhas, que determinam elevada queda de botões florais, flores e frutos em desenvolvimento, que é tanto mais intensa quanto maior o índice de floração (Agustí \& Almela, 1991). 
Existe uma relação inversa entre a velocidade de crescimento do fruto ou tamanho inicial do ovário e probabilidade de abcisão de frutos (Zucconi et al., 1978). Todos os fatores que estimulam o crescimento inicial do ovário aumentam a fixação de frutos.

Segundo Guardiola et al. (1988), as interações entre o número de frutos, o crescimento vegetativo das plantas e a queda natural precoce de frutos determinam o crescimento dos frutos nos estágios posteriores. A presença da folha é importante para a fixação de frutos, pelo seu papel de supridor de metabólitos (carboidratos, giberelinas e citocininas), tanto assim que a retirada de folhas, junto aos botões florais, reduz em $75 \%$ a fixação de frutos (Lenz, 1966).

A importância de um bom suprimento de nutrientes e hormônios, na fixação de frutos, é evidenciada pelos efeitos benéficos obtidos em diversos experimentos de anelagem da casca de ramos ou do tronco (Agustí \& Almela, 1991).

As flores e frutos são, portanto, considerados grandes consumidores de metabólitos. Por isso, quando a intensidade de floração é superior a 20 flores/100 nós do ramo, o índice de fixação de frutos diminui progressivamente (Becerra \& Guardiola, 1984).

A produção de frutos, em variedades de citros que florescem intensamente e cujos frutos não têm sementes, pode ser aumentada pela diminuição do índice de florescimento, reduzindo assim a competição entre flores e frutos, ou pelo incremento da fixação ou retenção de frutos.

Monselise \& Halevy (1964) observaram que duas aplicações foliares de $\mathrm{AG}_{3}$, na concentração de 10 ppm, por ocasião da indução floral, inibiram a floração em laranjeiras. As pulverizações de $\mathrm{AG}_{3}$, entretanto, devem ser feitas durante a indução floral, bem antes que as gemas iniciem a brotação, porque depois que já estiverem diferenciadas o $\mathrm{AG}_{3}$ não reduz mais o número de flores. Moss (1970) também recomenda duas pulverizações consecutivas, com 10 a 25 ppm de $\mathrm{AG}_{3}$ e 1\% de óleo mineral emulsionável, antes da diferenciação das gemas, para diminuir a intensidade da floração em plantas cítricas. Quanto às épocas exatas de aplicação devem ser realizados testes em cada região e com cada cultivar ou espécie.

Por sua vez, quando aplicado durante a floração (Moss,1972) ou no final da queda das pétalas (Agustí
\& Almela, 1991, em cultivares de frutos sem sementes como a tangerineira 'Clementina', o $\mathrm{AG}_{3}$, na dose de 10 ppm, aumenta a fixação de frutos. Esse efeito em geral é decorrente da aceleração do crescimento dos frutos de menor diâmetro, fazendo com que eles superem o tamanho crítico abaixo do qual ocorreria a abcisão durante a fase de queda natural (Agustí et al., 1982).

Todavia, o incremento da fixação de frutos, pela aplicação de $\mathrm{AG}_{3}$, pode não se refletir em aumento do número de frutos retidos na planta até a colheita (Moss, 1972), porque em algumas cultivares de citros o incremento da fixação inicial de frutos pode redundar em maior competição entre eles, por nutrientes, resultando em retardamento ou aumento da queda posterior de frutos (Krezdorn \& Brown, 1970).

A absorção do $\mathrm{AG}_{3}$ pela casca de frutas cítricas é favorecida pelo aumento da temperatura, na faixa de 5 a $35^{\circ} \mathrm{C}$, pela acidificação da solução até pH 4,0, pela adição de surfactantes e pelo aumento da umidade relativa até próximo do ponto de saturação (Greenberg \& Goldschmidt, 1989). A adição de surfactantes ou espalhantes adesivos às soluções de pulverização foliar duplicou o efeito do $\mathrm{AG}_{3}$, na conservação da cor verde de pomelos, em testes de laboratório (Coggins Junior \& Henning, 1989).

Para controlar o excesso de flores em laranjeiras de umbigo na Espanha, Agustí \& Almela (1991) recomendam a pulverização foliar com 10 ppm de $\mathrm{AG}_{3}$ e 15 ppm de 2,4-D em fins de novembro ou início de dezembro. Para aumentar a fixação de frutos eles recomendam uma pulverização adicional de $5 \mathrm{ppm}$ de $\mathrm{AG}_{3}$ quando $90 \%$ das flores perderem as pétalas.

Este trabalho teve por objetivo estudar o efeito da anelagem da casca e de pulverizações foliares de ácido giberélico, com e sem óleo mineral emulsionável, sobre a frutificação precoce de laranjeiras de umbigo 'Monte Parnaso'.

\section{MATERIALE MÉTODOS}

Foram realizados dois experimentos com laranjeiras 'Monte Parnaso' (Citrus sinensis Osbeck) com 2 anos de idade, enxertadas sobre Poncirus trifoliata, plantadas num espaçamento de 2,5 × 6,0 metros, em pomar situado no município de Butiá, Estado do Rio Grande do Sul.

O delineamento experimental foi em blocos ao acaso, com parcelas subdivididas, usando três plantas úteis, uni- 
formes e consecutivas, por subparcela, e quatro repetições. Entre cada subparcela, na linha de plantio, foram utilizadas duas árvores como bordadura.

Os tratamentos principais compreenderam duas dosagens de óleo mineral emulsionável: 0 e $1 \%$, do produto comercial Triona, em combinações com quatro concentrações de $\mathrm{AG}_{3}$. Os subtratamentos incluíram plantas que não foram submetidas à anelagem da casca e plantas com incisão anelar no tronco, $5 \mathrm{~cm}$ abaixo dos ramos iniciais da copa. Os subsubtratamentos constaram de quatro concentrações de ácido giberélico $\left(\mathrm{AG}_{3}\right)$, produto comercial Pro-Gibb): 0, 10, 20 e $30 \mathrm{ppm}$ de $\mathrm{AG}_{3}$. No experimento 1 as plantas sofreram duas pulverizações consecutivas de $\mathrm{AG}_{3}$ com óleo mineral emulsionável, com intervalo de sete dias, respectivamente em 22 e 29/07/93, aproximadamente 20 dias antes do início da brotação primaveril. No experimento 2 as plantas foram pulverizadas, com as mesmas doses de ácido giberélico e óleo mineral emulsionável, uma só vez, na plena floração, em 15/09/93.

Em ambos os experimentos, a anelagem da casca do tronco das árvores foi realizada logo após o final da floração, em 29/09/93, fazendo uma incisão anelar $\left(360^{\circ}\right)$ com uma tesoura de lâminas encurvadas para dentro, aplicando pressão apenas suficiente para cortar a casca, sem danificar o lenho. Essa incisão anelar da casca foi executada no final da floração com o objetivo de interromper temporariamente o movimento descendente da seiva elaborada, retendo-a por alguns dias na copa, para nutrir melhor os frutos e assim acelerar o seu crescimento e dimunuir a abcisão por ocasião da queda natural.

Os demais tratos culturais, tais como podas, adubações e controle de ervas daninhas, pragas e moléstias, foram uniformes em ambos os experimentos.

Para avaliação do efeito dos tratamentos foi realizada a contagem e pesagem dos frutos colhidos por planta, em agosto de 1994. Os dados obtidos foram submetidos à análise de variância e as médias foram comparadas pelo teste Tukey a $5 \%$.

\section{RESULTADOS E DISCUSSÃO}

Algumas plantas submetidas à anelagem evidenciaram clorose foliar dois a quatro meses após o tratamento, provavelmente porque o corte foi demasiado profundo, atrasando a cicatrização. Decorridos cinco a seis meses após a anelagem, essa clorose desapareceu totalmente, sem maiores conseqüências.

Não foram percebidos sintomas visuais de toxidez relativas aos tratamentos com $A_{3}$ e óleo mineral emulsionável.
A análise de variância não revelou efeito significativo dos tratamentos sobre o peso da produção total de frutos. Também não revelou interação entre os efeitos do $\mathrm{AG}_{3}$ com a anelagem da casca do tronco, nem com o uso do óleo mineral emulsionável.

$\mathrm{O}$ ácido giberélico, quando aplicado sucessivamente em 22 e 29/07/93, no experimento 1, exerceu um efeito linear de diminuição do número de frutos produzidos, em função do aumento das concentrações (Fig. 1). Esse efeito depressivo indica provável inibição da indução de gemas florais, exercida pelo $\mathrm{AG}_{3}$, diminuindo o número de flores e de frutos produzidos (Monselise \& Halevy, 1964).

É possível que o efeito de diminuição da frutificação exercido pelo $\mathrm{AG}_{3}$ fosse mais intenso se a aplicação tivesse sido antecipada para fins de maio ou início de junho, porque Agustí \& Almela (1991), na Espanha (Hemisfério Norte) recomendam a aplicação desse regulador de crescimento em fins de novembro a início de dezembro, que correspondem aos meses de maio e junho no Hemisfério Sul.

Considerando os efeitos de raleio de flores, $\mathrm{o}_{\mathrm{AG}_{3}}$ aplicado em 22 e 29/07/93 deveria ter diminuído a competição das flores e frutos por metabólitos, au-

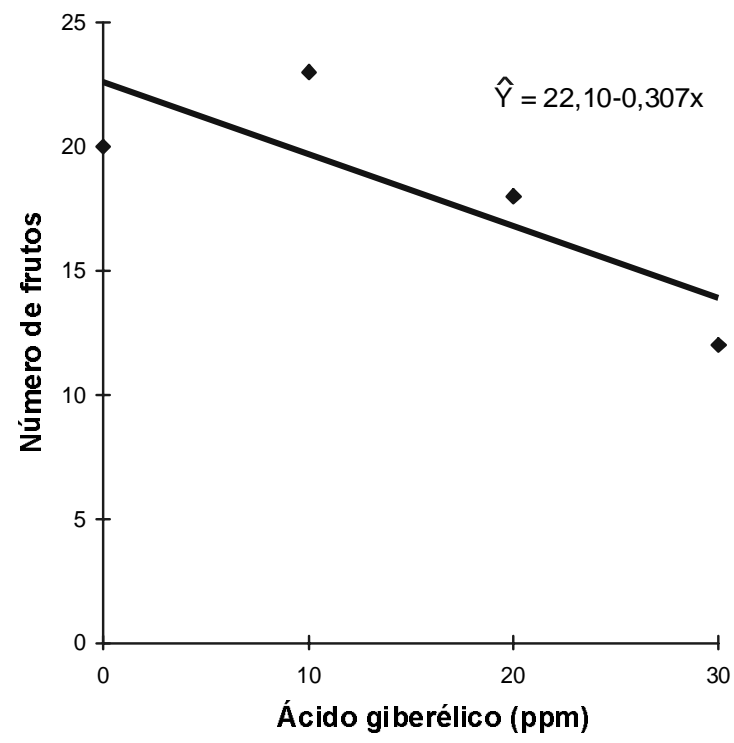

FIG. 1. Número de frutos produzidos por laranjeira de umbigo 'Monte Parnaso' aos três anos de idade, em função de pulverizações com quatro concentrações de ácido giberélico, em 22 e 27/07/93. 
mentando a retenção de frutos na planta (Zucconi et al., 1978). Isto, entretanto, não acontece em plantas com índice baixo de floração, inferior a 20 flores/100 nós (Becerra \& Guardiola, 1984), como ficou evidenciado neste trabalho. Na época de aplicação do $\mathrm{AG}_{3}$ as árvores estavam com apenas dois anos de idade e, conseqüentemente, com floração escassa. Por isso, inibindo a floração, neste caso, o $\mathrm{AG}_{3}$ diminuiu ainda mais o número de flores e frutos produzidos.

Quando aplicado em 15/09/93, no entanto (na ple-

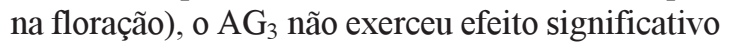
sobre o número de frutos produzidos (Tabela 1). Esse resultado aparentemente contraria a recomendação

TABELA 1. Efeito da dosagem de ácido giberélico $\left(\mathrm{AG}_{3}\right)$, pulverizado em 15/09/93 e da anelagem da casca do tronco, sobre o número de frutos produzidos por laranjeira de umbigo 'Monte Parnaso', aos três anos de idade (Experimento 2).

\begin{tabular}{ccc}
\hline $\begin{array}{c}\text { Doses de } \mathrm{AG}_{3} \\
(\mathrm{ppm})\end{array}$ & Sem anelagem & Com anelagem \\
\hline & --------- Número de frutos --------- \\
0 & 12,4 & 19,4 \\
10 & 14,1 & 14,6 \\
20 & 12,3 & 16,9 \\
30 & 12,6 & 16,1 \\
\hline
\end{tabular}

de pulverização com 5 ppm de $A_{3}$, no final da floração, feita por Agustí \& Almela (1991); contudo nem essa época de aplicação nem as doses de $\mathrm{AG}_{3}$ testadas foram as recomendadas pelos referidos autores. Além disso, tratando-se de plantas novas, ainda com floração escassa e intenso crescimento vegetativo, a maior ou menor fixação de frutos poderia estar mais relacionada a outros fatores, como a competição exercida pela brotação primaveril, do que propriamente pela disponibilidade de $\mathrm{AG}_{3}$.

Não foram observados efeitos significativos dos tratamentos com $\mathrm{AG}_{3}$ sobre o peso médio dos frutos produzidos.

Quanto ao efeito da anelagem da casca do tronco, no experimento 1 ela aumentou o número de frutos produzidos, independentemente da aplicação ou não de óleo mineral emulsionável (Tabela 2). Esse efeito favorável da anelagem da casca sobre a produção dos citros já foi evidenciado em muitos experimentos (Agustí \& Almela, 1991), devendo-se à retenção de metabólitos na copa, em benefício da melhor nutrição dos frutos, na fase crítica do início do desenvolvimento, logo após o florescimento (Zucconi et al., 1978). Entretanto, no experimento 2 a anelagem da casca do tronco somente aumentou a produção de frutos na ausência da aplicação de óleo mineral emulsionável (Tabela 2). Esse comportamento, um pouco distinto nos dois experimentos deve estar relacionado à época de aplicação do óleo, que no experimento 1 foi por ocasião da indução floral e

TABELA2. Número de frutos produzidos por laranjeira de umbigo 'Monte Parnaso' aos três anos de idade, em função de pulverizações com óleo mineral emulsionável, com e sem anelagem da casca do tronco, em dois experimentos ${ }^{1}$.

\begin{tabular}{|c|c|c|c|c|}
\hline \multirow[t]{2}{*}{ Experimento } & \multirow[t]{2}{*}{ Anelagem da casca } & \multicolumn{2}{|c|}{ Dosagem de óleo mineral } & \multirow[t]{2}{*}{ Média } \\
\hline & & $0 \%$ & $1 \%$ & \\
\hline & & \multicolumn{3}{|c|}{ - no de frutos - } \\
\hline Experimento 1 & Com anelagem & 24,50 & 14,50 & $19,50 \mathrm{~A}$ \\
\hline Experimento 1 & Sem anelagem & 19,83 & 11,17 & $15,50 \mathrm{~B}$ \\
\hline Média & & $22,17 \mathrm{a}$ & $12,84 b$ & \\
\hline Experimento 2 & Com anelagem & $16,94 \mathrm{a}$ & $15,31 \mathrm{a}$ & 16,13 \\
\hline Experimento 2 & Sem anelagem & $10,75 b$ & $15,94 \mathrm{a}$ & 13,35 \\
\hline Média & & 13,84 & 15,63 & \\
\hline
\end{tabular}

1 Médias seguidas de letras distintas, minúsculas nas linhas e maiúsculas nas colunas, diferiram entre si pelo teste de Duncan, a $5 \%$ de probabilidade. 
no experimento 2 ao final da floração. Neste caso o óleo pode ter contribuído para aumentar a queda natural de frutos (queda fisiológica), fazendo com que eles, fixados em menor número, fossem melhor nutridos, crescessem com maior rapidez e conseqüentemente fossem retidos com mais força pelas plantas, diminuindo a proporção de quedas em épocas posteriores (Agustí \& Almela, 1991).

$\mathrm{Na}$ Tabela 3 verifica-se que a anelagem da casca diminuiu o peso dos frutos. Isso é uma desvantagem em mercados nos quais os frutos grandes são mais valorizados. Ademais, como não houve efeito sobre o peso da produção, a diminuição do tamanho dos frutos pode ser favorável para mercados que preferem frutos menores.

Quanto ao efeito do óleo mineral emulsionável, as duas pulverizações com $1 \%$ em fins de julho (experimento 1) diminuíram a produção de frutos em aproximadamente $42 \%$, independentemente do uso da anelagem ou não da casca do tronco (Tabela 2). Quando foi realizada uma só aplicação de óleo mineral na plena floração, não foram detectados efeitos significativos. Verificou-se também que o óleo mineral não afetou o peso médio dos frutos (Tabela 4).

TABELA3. Peso médio de laranjas 'Monte Parnaso' em função da anelagem da casca do tronco feita uma semana após a floração, em dois experimentos ${ }^{1}$.

\begin{tabular}{lcc}
\hline Anelagem da casca & \multicolumn{2}{c}{ Peso médio de frutos $(\mathrm{g})$} \\
\cline { 2 - 3 } & Experimento 1 & Experimento 2 \\
\hline Sem anelagem & $310,2 \mathrm{a}$ & $305,6 \mathrm{a}$ \\
Com anelagem & $271,1 \mathrm{~b}$ & $263,4 \mathrm{a}$ \\
\hline $\begin{array}{l}1 \\
\text { Médias seguidas de letras distintas na coluna diferem entre si pelo teste } \\
\text { de Duncan a 5\% de probabilidade. }\end{array}$
\end{tabular}

TABela 4. Peso médio de laranjas 'Monte Parnaso' em função da adição de óleo mineral emulsionável a pulverizações com ácido giberélico em dois experimentos.

\begin{tabular}{ccc}
\hline \multirow{2}{*}{$\begin{array}{c}\text { Dosagem de } \\
\text { óleo mineral }\end{array}$} & \multicolumn{2}{c}{ Peso médio de frutos $(\mathrm{g})$} \\
\cline { 2 - 3 } & Experimento 1 & Experimento 2 \\
\hline $0 \%$ & 276,5 & 292,0 \\
$1 \%$ & 304,5 & 276,9 \\
\hline
\end{tabular}

Outros pesquisadores já haviam observado, em laranjeiras de umbigo 'Washington Navel', que aplicações de óleo mineral a $1 \%$ exerceram uma redução da floração de $20 \%$; quando associadas com 25 ppm de $\mathrm{AG}_{3}$, a inibição da floração foi total (Moss, 1973). A ação inibidora da floração pelo óleo foi atribuída principalmente à presença de detergentes que exerceriam efeito sinergético com o $\mathrm{AG}_{3}$. Outras duas hipóteses podem ser levantadas para explicar o fato. A primeira, de que o óleo mineral emulsionável possa exercer efeito fitotóxico ou de diminuição das trocas gasosas $\left(\mathrm{CO}_{2} / \mathrm{O}_{2}\right)$ nos estômatos, dificultando a fotossíntese. Em conseqüência, pode ter ocorrido a inibição da formação de gemas florais ou de frutos, resultantes de menor disponibilidade de fotossintatos. Uma segunda hipótese, que não exclui a primeira, pode residir no fato de que duas pulverizações consecutivas com intervalo de apenas uma semana, feitas em fins de julho, podem ter elevado o teor do óleo mineral ao nível fitotóxico (porém sem sintomas visuais), afetando mecanismos vitais para o florescimento e frutificação da laranjeira 'Monte Parnaso', de modo muito mais acentuado do que em uma só aplicação feita na plena floração, pois aplicado nessa fase o óleo mineral poderia ter exercido efeito de raleio de frutos.

Em novos experimentos é importante estudar o efeito do óleo mineral sobre a frutificação dos citros, principalmente porque se trata de um produto recomendado para o controle de cochonilhas e que pode diminuir a produção de citros quando usado de forma inoportuna em cultivares sem sementes. Além do mais, o baixo custo do óleo mineral emulsionável pode abrir novas perspectivas de uso para regular a frutificação em citros.

\section{CONCLUSÕES}

1. Pulverizações no final de julho, 20 dias antes da brotação primaveril, com dosagens crescentes de ácido giberélico de 0 a $30 \mathrm{ppm}$, diminuem linearmente o número de frutos produzidos por árvore; pulverizações de ácidos giberélico na plena floração não afetam a frutificação.

2. Óleo mineral a $1 \%$, aplicado em julho, diminui em aproximadamente $42 \%$ o número de frutos produzidos por árvore. 
3. A anelagem aumenta o número de frutos produzidos por árvore e diminui o peso médio dos frutos.

\section{REFERÊNCIAS}

Agustí, M.; AlMELA, V. Aplicación de fitorreguladores en citricultura. Barcelona: Aedos, 1991. 169p.

AGUSTÍ, M.; GARCÍA-MARY, F.; GUARDIOLA, J.L. Gibberellic acid and fruit set in sweet orange Scientia Horticulturae, v.17, p.257-264, 1982.

BECERRA, S.; GUARDIOLA, J.L. Inter-relationship between flowering and fruiting in sweet orange, cultivar Navelina. In: INTERNATIONAL CITRUS CONGRESS, 5., 1984, São Paulo. Proceedings... São Paulo: International Society of Citriculture, 1984. v.1, p.190-194.

COGGINS JUNIOR, C.W.; HENNING, G.L. Grapefruit rind blemish caused by interaction of Gibberellic Acid and wetting agents. In: INTERNATIONAL CITRUS CONGRESS MIDDLE EAST, 6., 1988, Tel Aviv. Proceedings... Tel Aviv: International Society of Citriculture, 1989. v.1, p.333-339.

GREENBERG, J.; GOLDSCHMIDT, E.E. The effectiveness of $\mathrm{AG}_{3}$ application to citrus fruit. In: INTERNATIONAL CITRUS CONGRESS MIDDLE EAST, 6., 1988, Tel Aviv. Proceedings... Tel Aviv: International Society of Citriculture, 1989. v.1, p.339-343.

GUARDIOLA, J.L.; ALMELA, V.; BARRÉS, M.T. Dual effect of auxins on fruit growth in 'Satsuma' mandarin. Scientia Horticulturae, Amsterdam, v.34, p.228-237, 1988.
KOLLER, O.C. Laranjeira de umbigo: aumento de produtividade. Jornal do Comércio, Porto Alegre, 23 de dez., 1993. p.4.

KREZDORN, A.H.; BROWN, H.D. Increasing yields of 'Minneola', 'Robinson' and 'Osceola' varieties with gibberellic acid sprays and girdling. Proceedings of the Florida State Horticultural Society, v.83, p.29-34, 1970.

LENZ, F. Flower and fruit development in Valencia Late orange as affected by type of inflorescence and nutritional status. Horticultural Research, Amsterdam, v.84, p.141-146, 1966.

MONSELISE, S.P.; HALEVY, A.H. Chemical inhibition and promotion of citrus flower bud induction. Proceedings of the American Society for Horticultural Science, Geneva, v.84, p.141-146, 1964.

MOSS, G.I. Chemical control of flower development in sweet orange. Australian Journal of Agricultural Research, Victoria, v.21, p.233-242, 1970.

MOSS, G.I. New methods of crops thinning for citrus. In: CONGRESSO MUNDIAL DE CITRICULTURA, 1., 1973, Murcia. Anales... Murcia: International Society Citriculture, 1973. v.12, p.243-247.

MOSS, G.I. Promoting fruit-set and yield in sweet orange. Australian Journal of Experimental Agriculture and Animal Husbandry, Melbourn, v.114, p.96$-102,1972$.

ZUCCONI, F.; MONSELISE, S.P.; GOREN, R. Growth abscission relationships in developing orange fruit. Scientia Horticulturae, Amsterdam, v.9, p.137$-146,1978$. 